
\title{
Resesarch Suluare \\ Engineering of AAV-Mediated in Vivo Targeted DNA Methylation Editing System via Staphylococcus Aureus Derived Cas9-SunTag
}

Naoki Yamamoto

Hoshi University: Hoshi Yakka Daigaku

Sumiyo Morita

Gunma University: Gunma Daigaku

Izuho Hatada

Gunma University: Gunma Daigaku

Maky Ideta-Otsuka

Hoshi University: Hoshi Yakka Daigaku

Hideki Tamura

Hoshi University: Hoshi Yakka Daigaku

Minoru Narita

Hoshi University: Hoshi Yakka Daigaku

Katsuhide Igarashi ( $\nabla$ k-igarashi@hoshi.ac.jp )

Hoshi University

Research

Keywords: Targeted DNA Methylation, Epigenome Editing, Epigenome Therapy, AAV, SaCas9, Bdnf, SunTag

Posted Date: November 16th, 2021

DOI: https://doi.org/10.21203/rs.3.rs-1065603/v1

License: (a) (1) This work is licensed under a Creative Commons Attribution 4.0 International License. Read Full License 


\section{Abstract}

DNA methylation aberrations have been implicated in the pathogenesis of various psychiatric and developmental disease states. However, basic research and therapeutic in vivo applications targeting aberrant DNA methylation are still limited due to the lack of targeted DNA methylation editing systems that work on capacity-limited adeno-associated virus (AAV), a highly versatile in vivo gene delivery vector. Here, we report the engineering of AAV-based vector system for in vivo targeted DNA methylation/demethylation by integrating small Cas 9 orthologues (SaCas9) and a dCas9-SunTag methodology. In this system, dSaCas9-SunTag targeting protein and anti-SunTag-mini-antibody (scFv) fused effector protein were packaged into different vectors for efficient in vivo delivery by AAV. We demonstrated that co-introduction of AAV vectors carrying dSaCas9-SunTag and scFv-fused Dnmt3A, Dnmt3L, or Dnmt3A-Dnmt3L fusion proteins induced robust DNA methylation on the Bdnf gene locus in a guide RNA dependent manner in vitro. The scFv-fused TET1 and TET2 successfully induced robust targeted DNA demethylation in the H19 ICR. We also constructed a doxycycline (Dox)-inducible target DNA methylation system utilizing dSaCas9-SunTag and scFv-Dnmt3AL. Finally, we showed that AAVmediated delivery of dSaCas9-SunTag system induced DNA methylation on the Bdnf gene locus and suppressed activity-dependent but not basal $B d n f$ expression after contextual fear learning in the Hippocampus of adult mouse. Our in vivo targeted DNA methylation editing system can be a promising platform for understating the causal relationship between psychiatric disease and aberrant DNA modifications.

\section{Introduction}

Aberrant DNA methylation has been widely observed in various psychiatric diseases, such as schizophrenia and major depression $[1,2,3,4,5,6]$. A growing body of research has been aimed at understating the biological function of the local epigenetic status via targeted manipulation of local epigenetic status $[7,8,9,10,11]$. Many groups have reported successful attempts of targeted manipulation of local DNA methylation status, called targeted DNA methylation editing, using a fusion protein containing a DNA targeting module and a catalytic domain of DNA methylation modifier $[9,11,12$, $13,14,15,16,17,18,19,20,21,22,23,24]$. Using targeted DNA methylation editing methodology, the causal relationship between aberrant DNA methylation and abnormal expression disease-related gene was examined in vitro $[16,18,19,21,24,25]$. A few studies have reported successful in vivo targeted DNA methylation editing by using lentivirus vectors $[17,18]$, whereas adeno-associated virus (AAV)-based targeted DNA methylation has not yet been reported.

AAV-based targeted DNA methylation editing offers several advantages over a lentivirus-based methodology for in vivo application. First, AAV vectors possess higher affinity for neuronal tissue and can induce transgene expression in a broader region [26]. Second, AAV vectors have lower pathogenicity than lentivirus vectors, as AAVs require another helper virus to mediate their replication $[27,28]$. Third, lentivirus vectors are randomly integrated into the host cell genome, whereas AAV vectors minimize this possibility, which can in turn reduce the possibility of insertional effects $[28,29]$. For these reasons, there are many 
on-going clinical trials using AAV targeting Parkinson's disease, Alzheimer's disease, and Epilepsy [30]. Nevertheless, AAV-based targeted DNA methylation editing is hampered by the restrictive cargo size of AAV (4.7 kb of transgene sequence) and bigger size of a fusion protein with catalytically inactive Streptococcus pyogenes derived Cas9 (dCas9, $4.2 \mathrm{~kb}$ ) [31], widely used targeting module, and effector DNA methylation domain (about 1-2 kb) [11, 18, 19, 20, 21, 22, 23].

Aiming to develop AAV-based targeted DNA methylation editing systems, we focused a new targeted methylation editing system using dCas 9 fused with a GCN4 peptides repeat sequence (SunTag) [17]. In the dCas9-SunTag system, multiple copies of anti-GCN4 antibody-fused effector domain were recruited to dCas9-SunTag protein on target gene locus [17, 32, 33]. By recruiting multiple copies of effector proteins, dCas9-SunTag system exhibits a much higher induction rate of DNA methylation/demethylation at target sites with greater specificity than other methylation editing systems that recruit only one dCas9-effector fusion protein to the target gene locus $[17,33]$. The dCas9-SunTag system offers another advantage for AAV-based targeted methylation editing; unlike a dCas9-effector direct fusion protein, it is easy for the dCas9-SunTag system to be split into smaller units, reducing the size of the transgene payloads within the AAV vectors. In this study, we employed smaller dCas 9 orthologues derived from Staphylococcus aureus (dSaCas9) as a targeting module [31]. Comparing previously used Streptococcus pyogenes derived dCas $9(4.2 \mathrm{~kb})$, the smaller dSaCas9 $(3.1 \mathrm{~kb})$ can maximize the number of fused SunTag units within the loading limit of AAVs $(4.7 \mathrm{~kb})$ and amplify the catalytic activity on the target locus by recruiting more copies of the antibody-fused effector protein. Furthermore, given the flexible vector design space of this splitting vector system, we examined the efficacy of various sizes of effector domains mediating DNA methylation or demethylation.

We report here the development of AAV vector tool kits for targeted methylation editing via a combination of dSaCas9-SunTag and methylation/demethylation domain [17, 24, 32]. We separately induced vectors expressing dSaCas9 labeled with three SunTag peptides (dSaCas9-SunTag) and a catalytic domain of de novo DNA methyltransferase 3a (Dnmt3ACD) or the C-terminal domain of DNA methyltransferase 3-like (Dnmt3LCD) or a fusion protein with Dnmt3ACD and Dnmt3LCD (Dnmt3AL) fused to a mini-antibody specific for SunTag (scFv). Co-induction of dSaCas9-SunTag and each scFv-Dnmt3ACD or scFvDnmt3LCD or scFv-Dnmt3AL could promote DNA methylation on the targeted Bdnf locus in a guide RNA dependent manner. Specifically, scFv-Dnmt3AL showed highest methylation efficiency. We also developed a targeted demethylation system using the catalytic domain of TET1 or TET2 (scFv-TET1CD, scFv-TET2CD). Both scFv-TET1CD and scFv-TET2CD induced robust DNA demethylation at the target locus. Furthermore, we showed that Dox-induced temporal expression of dSaCas9-SunTag and scFvDnmt3AL could induce DNA methylation at the Bdnf gene locus. Finally, we demonstrated that intracranial injection of an AAV cocktail loading a targeted DNA methylation system to the mouse dorsal hippocampus (HPC) suppressed activity-dependent expression of $B d n f$ but not basal expression in adult mouse HPC.

\section{Results}


We generated a dSaCas9-SunTag mediated targeted DNA methylation system mediated by multiple AAV vectors (Fig. 1a-d). The first AAV vector contained dSaCas9 fused with SunTag $_{3 \mathrm{x}}$ which consisted of three tandem arrays of GCN4 peptides (dSaCas9-SunTag ${ }_{3 x}$, SCP3) under the control of the cytomegalovirus (CMV) promoter (Fig. 1a). We employed the optimized length (22 amino acids) for the linker separating each GCN4 peptide unit in the array [17], and optimized locations of the nuclear localization signal (NLS) for improved efficiency (Fig. 1a) [18]. The second AAV vector contained sgRNA under the control of the human U6 promoter, reverse tetracycline-controlled transactivator (rtTA), and red fluorescent tdTomato connected with a self-cleavable peptide (P2A) (Fig. 1b). A third AAV vector contained anti-GCN4 minimum antibody fused with either the catalytic domain of mouse Dnmt3a (scFv-Dnmt3ACD) or the C-terminal domain of Dnmt3I (scFv-Dnmt3LCD) or Dnmt3ACD-Dnmt3LCD fusion proteins (scFV-Dnmt3AL) under the control of the CMV promoter (Fig. 1c). Considering previous reports about the in vitro targeted DNA methylation system using Dnmt3ACD [13, 33, 34], we prepared scFv-Dnmt3ACD. The C-terminal domain of Dnmt3I is enzymatically inactive but required for the de novo DNA methylation of imprinted genes via interaction with dimerized Dnmt3I and Dnmt3a [35, 36]. Because enzymatically inactive Dnmt3LCD might reduce potential off-target effects, we also prepared scFv-Dnmt3LCD. Based on a previously reported in vitro targeted DNA methylation system using the Dnmt3ACD-Dnmt3LCD fusion proteins [34], we generated an AAV vector expressing scFv-Dnmt3AL. Because the Dnmt3ACD-Dnmt3LCD fusion protein shows about 10-fold higher de novo DNA methylation activity than Dnmt3ACD in vitro [15], scFvDnmt3AL was expected to have highest DNA methylation efficiency. These systems consisting of dSaCas9-SunTag ${ }_{3 x}$ and scFv-Dnmt3ACD or scFv-Dnmt3LCD or scFv-Dnmt3AL, were termed SCP3-A, SCP3-L and SCP3-AL, respectively. We designed three sgRNAs targeting CpGs around the promoter of $B d n f$ exon 4 to determine whether our system can induce DNA methylation on this promoter (Fig. 1e). This region is one of the more well-known key disease-related genomic loci involved in neural activitydependent transcriptional regulation of $B d n f$ genes via their local DNA methylation profile [18]. Forty-eight hours after transfection of the three sgRNAs and the SCP3-A, SCP3-L or SCP3-AL into N2A cells, tdTomato-expressing cells were selected by fluorescence-activated cell sorting (FACS) and cultured for another 4 days (Fig. 1f). DNA and RNA were extracted for quantitative bisulfite pyrosequencing and quantitative PCR (qPCR) 6 days after transfection, respectively. These cells expressed dSaCas 9 and scFvfused effector genes (Fig. 1g). Around $20 \%$ of $\mathrm{CpGs}$ at the promoter of $B d n f$ exon 4 were methylated in untreated negative control samples (WT) (Fig. $1 \mathrm{~h}$ right panel). In contrast, $38.4 \%$ and $44.5 \%$ of CpGs were methylated by SCP3-A and SCP3-L in the presence of targeting sgRNA mix but not the sgRNA control, respectively (Fig. 1h, i right panel; SCP3-A: control-sgRNA vs. Bdnf-sgRNA, ${ }^{\star *} p<0.01$, SCP3-L: controlsgRNA vs. Bdnf-sgRNA, ${ }^{\star \star \star \star} p<0.0001$ One-way ANOVA with post-hoc Tukey's multiple comparisons test). SCP3-L showed robust methylation than SCL3-A on almost all examined CpG sites, except for one CpG site located -108 bp upstream from TSS (Fig. 1h, i left panel). Beside a similar amount of the scFvfused effector protein was expressed in the control and target sgRNA groups, off-target DNA methylation activities derived from over-expressed scFv-Dnmt3ADC or scFv-Dnmt3LDC were not observed (Fig. 1h, i right panel; WT vs. SCP3-A \& control-sgRNA or SCP3-L \& control-sgRNA, not significant; One-way ANOVA with post-hoc Tukey's multiple comparisons test). Comparing to the DNA methylation levels induced by SCP3-A and SCP3-L, SCP3-AL achieved more efficient methylation on all examined CpG sites (Fig. 1j left 
panel), and higher average methylation level (65.6\%) without off-target DNA methylation activity derived from over-expressed scFv-Dnmt3AL (Fig. 1j right panel; SCP3-AL \& control-sgRNA vs. SCP3-AL \& BdnfsgRNA, SCP3-A \& Bdnf-sgRNA vs. SCP3-AL \& Bdnf-sgRNA, SCP3-L \& Bdnf-sgRNA vs. SCP3-AL \& BdnfsgRNA ****p < 0.0001; One-way ANOVA with post-hoc Tukey's multiple comparisons test). Because basal expression of Bdnf was quite low in N2A cells (Data not shown), we did not examine effects of targeted methylation editing on the $B d n f$ gene. From these results, we concluded that targeted methylation could be achieved using a novel combination of dSaCas9 and SunTag system, and the SCP3-AL system possesses higher DNA methylation efficiency than either SCP3-A and SCP3-L.

We also engineered an AAV-mediated targeted demethylation system. In previous reports, targeted DNA demethylation was achieved by a combination of dCas9-SunTag and the catalytic domain of human TET1 DNA demethylation catalyzing enzyme fused with anti-GCN4 minimum antibody (scFv-TET1CD) $[17,24]$. In addition to SCFv-TET1CD, we prepared a smaller effector domain, the catalytic domain of the human TET2 enzyme fused with scFv (scFv-TET2CD) (Fig. 2a). Referring to the crystal structure data of human TET2 [37], we cloned the minimum catalytic domains of human TET2 and connected them with a 15 bp linker sequence. The resulting scFv-TET2CD was 774 bp smaller than scFv-TET1CD. These targeted demethylation systems consisting of dSaCas9-SunTag ${ }_{3 x}$ and scFv-TET1CD or scFv-TET2CD were termed SCP3-TET1 and SCP3-TET2, respectively. To assess the DNA demethylation efficiencies of SCP3-TET1 and SCP3-TET2 in vitro, we designed five sgRNAs targeting the methylation-sensitive CTCFbinding sites harboring the H19 ICR locus (Fig. 2b). DNA and RNA were extracted from FACS-sorted cells 6 days after transfection of AAV constructs expressing the five sgRNAs targeting the H19 ICR locus and either SCP3-TET1 or SCP3-TET2 (Fig. 2c). The dSaCas9 gene and effector genes were expressed in sorted cells (Fig. $2 \mathrm{f}$ and $\mathrm{g}$ ). The $\mathrm{CpGs}$ of the targeted CTCF-binding site was highly methylated in untreated cells (81.8\%, Fig. 2d, e right panel). Both SCP3-TET1 and SCP-TET2 induced CpG demethylation on the targeted CTCF-binding site in an sgRNA-dependent manner (Fig. 2d and e right panel; SCP-TET1: control-sgRNA vs. H19-sgRNA, ${ }^{\star *} p<0.01$, SCP3-TET2: control-sgRNA vs. H19-sgRNA, ${ }^{\star * \star *} p<0.0001$; WT vs. control-sgRNA, not significant; One-way ANOVA with post-hoc Tukey's multiple comparisons test). Offtarget demethylation on the target site via overexpressing of ScFv-TET1 or scFv-TET2 gene was not observed on all examined CpG sites (Fig. 2d, e left panel; WT vs. control-sgRNA, not significant; Two-way ANOVA with post-hoc Tukey's multiple comparisons test). In addition, we observed significant upregulation of the $\mathrm{H} 19$ gene by SCP-TET2 (Fig. $2 \mathrm{~h}$ and i, SCP-TET2: control-sgRNA vs. H19-sgRNA, ${ }^{*} \mathrm{p}<0.05$, t-test). From these results, we concluded that the targeted DNA demethylation was also achieved by our dSaCas9-SunTag system.

As mentioned above, one of the therapeutic merits of targeted DNA manipulation technology is a langlasting effect on transcriptional regulation even after expression of the effector genes has ceased [21, 38]. From this point of view, we applied our targeted methylation system to a doxycycline (Dox)-inducible gene expression system. We generated dox-inducible AAV plasmids carrying dSaCas9-SunTag $3 x$ or scFvDnmt3AL, termed treSCP3-AL (Fig. 3a-c). We examined whether the treSCP3-AL system could induce targeted methylation in vitro on the promoter of Bdnf exon 4 after Dox-treatments. The same sgRNA set 
targeting Bdnf exon 4 was used (Fig. 3d). The tdTomato-expressing cells were sorted by FACS forty-eight hours after transfection of the sgRNAs and the components of treSCP3-AL into N2A cells. DNA and RNA were extracted after 4 days following culture in presence of Dox (Fig. 3e). Beside slight off-target methylation observed on a CpG site (Fig. $3 f$ left panel; -65 bp from TSS:WT vs. control-sgRNA * $p<0.05$; Two-way ANOVA with post-hoc Tukey's multiple comparisons test), on average the treSCP3-AL system induced a significant increase of methylation on the target locus in sgRNA dependent manner (Fig. $3 f$ right panel; WT vs. Bdnf-sgRNA, control-sgRNA vs. Bdnf-sgRNA, ${ }^{\star \star \star \star} p<0.0001$; WT vs. control-sgRNA, not significant; One-way ANOVA with post-hoc Tukey's multiple comparisons test). The methylation efficiency of dox-induced treSCP3-AL was comparable to that of SCP3-AL in which dSaCas9-SunTag ${ }_{3 x}$ and scFVDnmt3AL are controlled by CMV promoter (Fig. 3f right panel, treSCP3-AL \& Bdnf-sgRNA vs. SCP3-AL \& Bdnf-sgRNA, not significant; One-way ANOVA with post-hoc Tukey's multiple comparisons test).

Lastly, we investigated whether our targeted methylation system can be applied in vivo. For this purpose, we generated AAVs carrying the treSCP3-AL system showed in Fig. 3 and injected the AAV cocktails into the dorsal HPC of adult mice. We used the same sgRNA set targeting Bdnf exon 4 that was used for the in vitro studies (Fig. 3d). Expression of dSaCas9 and scFv-Dnmt3AL was induced by administration of Dox in drinking water for 4 days, followed by 7 days of rest (Fig. 4a). These animals were sacrificed for subsequent immuno-histochemical (IHC) and quantitative DNA methylation analysis (Fig. 4a). Expression of myc-tagged scFv-Dnmt3AL and tdTomato signal derived from sgRNA-expressing AAV was observed via IHC analyses of dox-administered dorsal HPC (Fig. 4b). We also confirmed strong expression of dSaCas 9 and scFV by qPCR analysis (Fig. 4c). These data clearly indicated that the components of treSCP3-AL were properly delivered into the adult mouse HPC via AAV. As a result, treSCP3-AL system successfully induced a significant increase of DNA methylation on the target locus in vivo (Fig. 4d right panel; WT vs. Bdnf-sgRNA, ${ }^{\star \star \star \star} p<0.0001$, control-sgRNA vs. Bdnf-sgRNA, ${ }^{* \star} p<0.001$; One-way ANOVA with post-hoc Tukey's multiple comparisons test). However, mild but significant off-target methylation by overexpression of scFV-Dnmt3AL was also observed (Fig. 4f right panel; WT vs. control-sgRNA **p $<0.01$; Two-way ANOVA with post-hoc Tukey's multiple comparisons test). Consistent with the previous reports that methylation level of $B d n f$ exon 4 was correlated with the activity-dependent expression of $B d n f$ [39, 40, 41], targeted methylation by treSCP3-AL did not affect basal expression of Bdnf in adult HPC (Fig. 4e). Previously, it was shown that contextual fear conditioning induced DNA hypomethylation within the Bdnf exon 4 promoter in the mouse and rat hippocampal CA1, and that this is correlated with the upregulation of $B d n f$ exon 4 mRNA expression [42, 43]. Based on these reports, we next examined whether the AAVmediated targeted DNA methylation on the adult mouse CA1 inhibited the activity-dependent transcriptional up-regulation of Bdnf gene after fear learning (Fig. 4f). After AAV injection, expression of dSaCas9 and scFv-Dnmt3AL was induced by administration of Dox in drinking water for following 7 days (Fig. 4f). 1 week after injection, animals explored a novel environment and were exposed to foot shock (Contextual fear conditioning, CFC). We repeated CFC to acquire robust activity-dependent $B d n f$ expression, and sacrificed animals $1 \mathrm{hr}$ after 2nd CFC (Fig. 4f). All animals showed robust freezing behavior after foot shocks (Fig. 4g). We also prepared AAV-injected controls that were never given a foot shock and taken from the home cage. The expression of dSaCas9-SunTag3x and scFV-Dnmt3AL were 
properly induced into the adult mouse CA1 via AAV (Fig. 4h). We observed up-regulation of Bdnf from animals after contextual fear learning compared to the inexperienced animals (Fig. 4i; con no CFC vs. con $1 \mathrm{hr}$ after $\mathrm{CFC},{ }^{*} \mathrm{p}<0.05$; One-way ANOVA with post-hoc Tukey's multiple comparisons test). The activity dependent up-regulation of $B d n f$ contextual fear learning was inhibited only in the presence of the sgRNA mix targeting the promoter of $B d n f$ exon 4 but not control sgRNA (Fig. 4i; mix no CFC vs. mix $1 \mathrm{hr}$ after CFC, not significant; con $1 \mathrm{hr}$ after CFC vs. mix $1 \mathrm{hr}$ after CFC, ${ }^{\star} \mathrm{p}<0.05$; One-way ANOVA with post-hoc Tukey's multiple comparisons test). Our in vivo and in vitro results strongly supported the conclusion that AAV-mediated induction of SCP3-AL and targeting sgRNAs into the adult mouse HPC inhibited the learning induced transcriptional up-regulation of the Bdnf gene via targeted DNA methylation and the successful development of Dox-inducible AAV-mediated in vivo targeted DNA methylation editing system.

\section{Discussion}

We here describe the engineering of an AAV-mediated in vivo targeted DNA methylation editing system with a combination of dSaCas 9 and SunTag system. In this system, we reduced the size of the transgene to the load capacity-limit of AAVs by splitting into two smaller vectors carrying the targeting domain and effector domain independently. Several groups reported new effector domain proteins using dCas9SunTag to accomplish more efficient and specific target DNA methylation editing including the functional domains of TET1, Dnmt3a and Dnmt3I [17, 22, 33, 44]. Given the flexible vector design space of this split vector system, we could examine the efficacy of various sizes of effector domains, such as scFVDnmt3LCD and scFV-Dnmt3AL, a fusion protein with Dnmt3ACD and Dnmt3LCD. We found scFVDnmt3AL showed significantly higher methylation efficiency than cFV-Dnmt3LCD or previously reported scFV-Dnmt3ACD (Fig. 1j). In addition, we also designed a novel effector domain, scFv-TET2, whose size is two-thirds of the previously reported scFv-TET1 and induced robust sgRNA-dependent DNA demethylation (Fig. 2e). The extensibility of our system could be a useful for assessing other effector domains in vivo with more robust and less off-target activity, such as ScFV-Dnmt3AL variants reported to reduced their endogenous DNA binding [44].

BDNF is widely produced in the cortex throughout life and is essential for normal physiological processes in the adult mammalian brain. For example, postmortem studies showed the downregulation of $B D N F$ expression in patients with schizophrenia and mood disorders, which are often associated with increased DNA methylation at the $B D N F$ promoter $[3,4,5]$. In animal experiments, infusions of a DNA methyltransferase inhibitor significantly altered DNA methylation around $B d n f$ exon 4 and triggered changes in exon specific $B d n f$ mRNA levels, indicating that altered DNA methylation is sufficient to drive expression of $B d n f$ both in vivo and in vitro $[42,45]$. The therapeutic effects of $B d n f$ were assessed in animal models. Viral vector-mediated gene delivery of $B d n f$ to the hippocampal region exhibits potent therapeutic effects including restoring disease-associated aberrant gene expression, prevention of cell death and improvements in learning and memory abilities in animal models of Alzheimer's disease [46, 47]. Compared to AAV-mediated gene delivery, our AAV-mediated epigenetic manipulation has several potential advantages towards the development of precision medicine. Firstly, targeted DNA manipulation technology has a long-lasting effect on the transcriptional regulation of target genes even after 
expression of the effector genes has ceased [21, 38]. Because the effector genes and dSaCas9 were under control of a dox-inducible promoter in our in vivo targeted methylation system, the off-target effects derived from dSaCas 9 and effector proteins can be minimized. Secondly, targeted epigenetic therapy can recover gene function by restoring the transcriptional regulation of the target gene in a diseased cell by restoring normal epigenetic patterning. On the other hand, AAV-mediated gene therapy aims to recover gene function with forced expression of the target gene under the control of an artificial promoter, perhaps a constitutively active promoter. Many biological processes require rigorous transcriptional timing of genes for their proper function. For example, precisely regulated oscillatory expression of key transcription factors for proper neuronal development $[48,49]$ and activity-dependent transcriptional regulation is important for spike-timing-dependent plasticity of neurons in our brain $[50,51]$. We showed that AAV-mediated delivery of the dSaCas9-SunTag targeted DNA methylation system induced DNA methylation on Bdnf gene locus and impaired activity-dependent expression of $B d n f$ but not basal expression in hippocampal HPC of adult mouse (Fig. 4). These results suggest that in vivo DNA methylation editing technology could be useful to normalize transcriptional regulation of target genes. Although, in vivo DNA methylation efficiency was still not particularly high (Fig. 4d), it could be improved by using the optimal promoter for in vivo expression of rtTA, such as the human Synapsin 1 (hSyn) promoter.

In summary, we successfully developed an AAV-mediated in vivo targeted DNA methylation system. We applied small Cas9 orthologues to the previously reported dCas9-SunTag epigenome editing system to address the difficulty in packaging a large dCas9 fusion protein required for targeted DNA methylation editing into an AAV delivery vehicle. Our dSaCas9-SunTag system induced both DNA methylation and demethylation on the target gene locus in an sgRNA-dependent manner in vitro. Furthermore, we showed that AAV-mediated delivery of the dox-inducible dSaCas9-SunTag and sgRNAs complex induced methylation on the Bdnf gene locus and specifically suppressed activity-dependent expression but not basal expression of Bdnf in the hippocampus of adult mouse. Based on our results, our dSaCas9-SunTag system appears promising for future applications of in vivo targeted DNA methylation editing-based therapy.

\section{Material And Methods}

\section{Cloning}

The CMV-dSaCas9-SunTag ${ }_{x 3}$ vector was cloned based on the Addgene plasmid \#61594 [52], in which 3x GCN4 sequence inserted after 3xHA tag by In-Fusion system (Takara). The 3x GCN4 sequence was cloned from Plasmid \#82560 [17]. The CMV-scFv-Dnmt3ACD, -Dnmt3LCD, -Dnmt3AL, -TET1CD and TET2CD vectors were cloned into a AAV vector backbone Addgene plasmid \#61594 [52], in which the dSaCas 9 sequence was replaced by each effector domain. The scFv sequence was cloned from Plasmid \#82559 [17]. The catalytic domain of mouse Dnmt3a (598-908 aa), C-terminal domain of mouse Dnmt3l (194-415 aa) and Dnmt3AL were cloned based on previous in vitro study [13,34]. Catalytic domains of human TET2 (1129-1936 and 1481-1843 aa) were cloned based on the reported structure [37] and 
connected with 15bp of GS linker sequence (GGGGSGGGGSGGGGS). The AAV vector co-expressing sgRNA rtTA and tdTomato was generated based on the Addgene plasmid \#61593 [52], in which the TBG promoter and dSaCas9 sequence were replaced by the CMV promoter and rtTA and tdTomato genes. The rtTA and tdTomato gene were connected with P2A peptide sequence. For Dox-inducible AAV vectors, CMV promoter were replaced by TRE promoter cloned from Addgene plasmid \#50916 [53]. The sequences of the sgRNAs were GTAATTCGTGCACTAGAGTGTC (Bdnf-1), CGAGGAGAGGACTGCTCTCGC (Bdnf-2), ACGCCTTCAGCGAGAAGCTCC (Bdnf-3), ATCAGTTGTGGGGTTTATACGC (H19-1), GACCCACAGCATTGCCATTT (H19-2), GTCACCCAAGTTCAGTACCTCAG (H19-3), GTGACCCCCCTGAGGTACTGAAC (H19-4), GCCGACCTTGTTGATTTGGGAGT (H19-5) and TGAGACCTGCCGTGGTCTCC (non-targeting control). The sequences of the sgRNAs were designed with the E-CRISP algorithm [54]. Non-targeting control sequence does not target any genome loci in mouse. All reagents described in this manuscript have been deposited with Addgene (plasmid IDs $177342-177365$, including CMV, hSyn or Dox-inducible promoter).

\section{Cell Culture Co-Transfections and FACS}

Neuro2A cells and AAVpro 293T cells (Takara) were cultivated at $37 \circ \mathrm{C}$ in a cell culture incubator with $95 \%$ relative humidity and $5 \%(\mathrm{v} / \mathrm{v})$ CO2. Cells were cultured in Dulbecco's modified Eagle's medium (DMEM, WAKO) supplemented with 10\% (v/v) fetal bovine serum (FBS, Gibco) and 1\% (v/v) Penicillin/Streptomycin (Sigma-Aldrich). Transfections of N2A cells were carried out at $80 \%$ confluency $24 \mathrm{~h}$ post-seeding in a 6 well dish using using polyethyleneimine MAX (PEI-MAX; $1 \mathrm{mg} / \mathrm{mL}$ in milliQ water, $\mathrm{pH}$ 7.2; Polysciences Inc., Warrington, PA, USA) as a transfection reagent following the manufacturer's

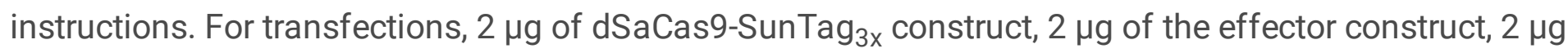
of the control sgRNA coding vectors construct or $500 \mathrm{ng}$ of three the sgRNA targeting Bdnf exon 4 or five the sgRNA targeting H19 ICR coding vectors were mixed. The medium was changed $12 \mathrm{hr}$ after transfection. Cells were harvested by trypsinization $36 \mathrm{~h}$ later and filtered through a $35 \mu \mathrm{m}$ pre-separation filter (Stellar Scientific) followed by preparative cell sorting by flow cytometry. The tdTomato-positive cells were collected using a FACSAria II (BD Bioscience). Dead cells and debris were discriminated with propidium iodide. The collected cells $\left(>5 \times 10^{\wedge} 4\right.$ cells) were cultured with 6 well dish for $48 \mathrm{hr}$ and harvested for further analysis. To examine the effect of the treSCP3-AL system, FACS sorted tdTomatopositive cells were cultured with Dox (10 ug/ml) for 3 days.

\section{Quantitative DNA Methylation Analysis}

The pyrosequencing technique was used for quantitative DNA methylation analysis. Total DNA was prepared from isolated tissues using the Allprep DNA/RNA mini kit (QIAGEN). $1 \mu \mathrm{g}$ of the purified DNA was used for bisulfite treatment with MethylEasy Xceed kit (Takara). The target DNA sequence was amplified using biotin-labeled one-sided PCR primers with 2x Gotaq2 G2 Hot Start Polumerase MasterMix (Promega). PCR conditions were as follows: 2 min at $98 \circ \mathrm{C}, 45$ cycles of $30 \mathrm{~s}$ at $98 \circ \mathrm{C}, 30 \mathrm{~s}$ at $50 \circ \mathrm{C}, 1$ min at $72 \circ \mathrm{C}$, and finally $10 \mathrm{~min}$ at $72 \circ \mathrm{C}$. Small aliquots from PCR products were subjected to $2 \%$ agarose gel electrophoresis for validation of specificity. The PCR products were used for pyrosequencing 
with PyroMarkGoldQ24 (QIAGEN) and analyzed PyroMarkQ24 software (QIAGEN) according to the manufacturer's instructions. The PCR primers: ATGGGAAAGTGGGTGGGAGTTTA and biotinACATCTACAAAACATACAATACCCTAAAA (Bdnf exon 4 \#1), AGGAtTGGAAGTGAAAAtATtTAtAAAGT and biotin-aCTaTCATATaATACCTCCTCTaCCT (Bdnf exon 4 \#2), biotin-GGAGGtATAAGAATTCTGtAAGGAG and ACATTTATAACCCCCCTAAAATACTA (H19-ICR). The sequence primers:

GGGAGTTTAAGAGAGGGTTTTA (Bdnf exon 4 \#1 sequence), tTAtAAAGtATGtAATGtttTGGA (Bdnf exon 4 \#2 sequence), CCAATACCAAAAATAAAAAAAC (H19-ICR sequence).

\section{Quantitative RT-PCR analysis.}

Total RNA was using the Allprep DNA/RNA mini kit (QIAGEN) with following DNase (Wako) treatment (15 min at $37 \circ \mathrm{C}, 10 \mathrm{~min}$ at $70 \circ \mathrm{C}$ ). $1 \mathrm{ng}$ of purified RNA was subjected to following reverse transcription (15 min at $37 \circ \mathrm{C}, 5 \mathrm{sec}$ at $85 \circ \mathrm{C}$ ) with PrimeScript RT Master Mix (Takara). Gene expression levels were measured with StepOnePlus ${ }^{\text {TM }}$ Real-Time PCR System (Applied Biosystems) using SYBR Premix Ex Taq II (Takara) according to the manufacturer's instructions. Gapdh was used as an inner control. The sequences of PCR primer were following. The PCR primers: TGTGTCCGTCGTGGATCTGA and CCTGCTTCACCACCTTCTTGA (Gapdh), GCTTCAGCCTGACCGACTAC and TACACGGTGTTCTTGCCGTT (scFv), AGCTGATCAACAAGAGCCCC and ATGGGCGTTCAGTTTGTTGC (dSaCas9), CAGGAGTACATATCGGCCACCA and GTAGGCCAAGTTGCCTTGTCCGT (Bdnf exon 4) and CACTGTATGCCCTAACCGCT and CCCAACCTCCCTCCCTAGAA (H19).

\section{Virus production and titration.}

For in-house viral production, AAVpro 293T cells (Takara) were maintained as described above in $150 \mathrm{~mm}$ plates. For each transfection, $8 \mu \mathrm{g}$ of pAAVdj serotype packaging plasmid, $8 \mu \mathrm{g}$ of helper plasmid, and $6 \mu \mathrm{g}$ of reconstructed AAV plasmid carrying the construct of interest mixed with $1000 \mathrm{~mL}$ of OPTI-MEM (Thermo) containing $48 \mu \mathrm{l}$ of PEI-MAX ( $1 \mathrm{mg} \mathrm{ml} / 1, \mathrm{pH} 7.2$ ) was prepared. After $10 \mathrm{~min}$ incubation, the mixture was applied to each dish. Medium was changed $12 \mathrm{hr}$ after transfection. Cells were harvested between $120 \mathrm{~h}$ post transfection by scraping and pelleting by centrifugation. The AAV2/dj viral particles were then purified with AAVpro Purification Kit (Takara). Purified viruses were titred by real-time qPCR using primer against AAV-ITR. AAVs with a titer of more than $3 \times 10^{\wedge} 11$ genome copy $/ \mathrm{ml}$ were used in following in vivo experiments.

\section{Animals}

Male C57BL/6J mice (8 weeks old, SLC) were used in this study. Animals were housed in the animal facilities at the School of Pharmacy and Pharmaceutical Sciences Hoshi University under specific pathogen-free conditions, maintained at $24^{\circ} \mathrm{C}$ on a $12 \mathrm{~h}$ light/dark cycle. Food and water were provided ad libitum. The animals were acclimatized for 1 week prior to the start of the experiments. All procedures were approved by the animal experiment ethics committee of Hoshi University and performed in accordance with the Hoshi University guidelines for the care and use of laboratory animals. 


\section{Mouse stereotaxic surgery}

Mice were anesthetized with $0.8 \%-1.5 \%$ isoflurane on a stereotaxic device. A small amount of $2 \%$ lidocaine was placed on and under the skin as a topical analgesic and a small hole was drilled above each injection site. Viruses were injected using a glass micropipette attached to a $1 \mathrm{ml} \mathrm{Hamilton}$ microsyringe through a microelectrode holder filled with mineral oil. A microsyringe pump and its controller were used to control the speed of the injection. The needle was slowly lowered to the target site and remained for $5 \mathrm{~min}$ after the injection. Viral injections were performed at a rate of $2 \mathrm{~nL} / \mathrm{second}$. Bilateral viral delivery into HPC of the WT male mice was targeted at AP: $-2.0 \mathrm{~mm}, \mathrm{ML}, \pm 1.5 \mathrm{~mm}, \mathrm{DV},-1.5$ $\mathrm{mm}$ from Bregma. WT mice were injected with $250 \mathrm{nl}$ of AAV cocktail (AAV2/dj-TRE-dSaCas9-SunTag ${ }_{\mathrm{x} 3}$ : AAV2/dj-TRE-scFv-Dnmt3AL : AAV2/dj-U6-sgRNA;CMV-rtTA-tdTomato mix=2:2:1) with $1 \%$ fast green dye (Sigma-Aldrich). An equal amount of three AAVs expressing sgRNA targeting the Bdnf exon 4 were mixed. At the end of surgery, mice were given intraperitoneal injections of $0.9 \%$ sterile saline and meloxicam (2 $\mathrm{mg} / \mathrm{kg}$ ) as an analgesic, placed on a heating pad until fully recovered from the anesthesia. After finishing all behavioral procedures, we performed histology to verify the target sites and expression of AAV injection. For DNA/RNA extraction, AAV-injected site on dorsal HPC labelled with fast green dye was manually isolated.

\section{Immunohistochemistry}

AAV injected mice were anesthetized using intraperitoneal injection of urethane $(1.25 \mathrm{~g} / \mathrm{kg})$ and transcardially perfused with phosphate-buffered saline (PBS; $\mathrm{pH} 7.4$ ) followed by $4 \%$ paraformaldehyde in PBS. Doxycycline $(1 \mathrm{mg} / \mathrm{ml})$ was administered with drinking water 4 days before perfusion. The brains were post-fixed overnight at $4{ }^{\circ} \mathrm{C}$ in the same fixative solution, transferred to $30 \%$ sucrose in PBS for $48 \mathrm{~h}$, and then cut into $30 \mu \mathrm{m}$ coronal sections using a CM1510-11 cryostat (Leica). The free-floating sections were blocked with $5 \%$ bovine serum albumin (BSA) and $0.3 \%$ Triton X-100 in PBS for $1 \mathrm{~h}$ at room temperature and incubated overnight at $4{ }^{\circ} \mathrm{C}$ with the following antibodies: mouse monoclonal anti-myc (1:200; 9B11, Cell Signaling). Alexa 488-conjugated donkey anti-mouse IgG (1:1,000; A21202, Thermo Fisher Scientific) was used for the following secondary antibody. Labeled sections were mounted onto microscope slides with Prolong Diamond Antifade Reagent containing DAPI (Thermo Fisher Scientific).

\section{Contextual fear conditioning}

Contextual fear conditioning was performed on WT male mice aged between 7 and 12 weeks during the light cycle. All animals were handled 5 minutes a day for 5 consecutive days prior to experimentation. A white plastic conditioning chamber $(17 \times 10 \times 10 \mathrm{~cm})$ was cleaned with water prior to the introduction of each individual mouse. Subject mice were kept in the conditioning chamber for total $270 \mathrm{~s}$. A foot-shock $(1 \mathrm{~s}, 0.3 \mathrm{~mA})$ was delivered at $120 \mathrm{~s}, 150 \mathrm{~s}, 180 \mathrm{~s}, 210 \mathrm{~s}$ and $240 \mathrm{~s}$. Mice remained in the conditioning chamber for a total of $270 \mathrm{~s}$. All sessions were video-recorded for automatic scoring of freezing (TimeFZ software, O'HARA \& Co., LTD.) which was defined as a period of immobility, lasting for at least $2 \mathrm{~s} .2^{\text {nd }}$ 
time $\mathrm{CFC}$ was performed after within $1 \mathrm{hr}$ after $1^{\text {st }} \mathrm{CFC}$. Half of $\mathrm{AAV}$-injected animals were subjected to CFC and sacrificed $1 \mathrm{hr}$ later. The remaining half was sacrificed without CFC (no CFC).

\section{Statistical analysis}

All data excluding Fig. $4 \mathrm{~g}$ are expressed as the mean \pm standard deviation. Fig. $4 \mathrm{~g}$ is expressed as the mean \pm standard error of the mean. Statistical analyses were performed using the Graphpad Prism 9 software. $P$ values of less than 0.05 were considered statistically significant. For all figures, $\mathrm{ns}=\mathrm{not}$

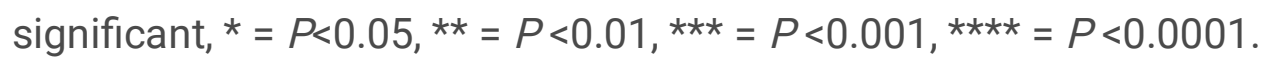

\section{Abbreviations}

AAV: Adeno-associated virus

Bdnf: Brain derived neurotrophic factor

Cas9: CRISPR associated protein

CFC: Contextual fear conditioning

CRISPR: Clustered regularly interspaced short palindromic repeat

dCas9: dead Cas9

Dox: Doxycycline

Dnmt: DNA methyltransferase

HPC: Hippocampus

hSyn: human Synapsin 1

ICR: Imprinting control region

ITR: Inverted terminal repeat

NLS: Nuclear localization signal

N2A: Neuro 2A

rtTA: Reverse tetracycline-controlled transactivator

sgRNA: Single guide RNA

SaCas9: Staphylococcus aureus derived smaller dCas9 
scFv: Single-chain variable fragment

Tet: Tet Methylcytosine Dioxygenase

\section{Declarations}

\section{Ethics approval and consent to participate}

The study was reviewed and approved by the animal experiment ethics committee of Hoshi University and performed in accordance with the Hoshi University guidelines for the care and use of laboratory animals.

\section{Consent for publication}

Not applicable.

\section{Availability of data and materials}

The datasets used and/or analyzed during the current study are available from the corresponding author on reasonable request. The authors plan to make the plasmids widely available to the academic community through Addgene.

\section{Competing interests}

The authors declare no competing financial interests.

\section{Funding}

We have been supported by Japan Society for the Promotion of Grant-in-Aid for Research Activity Start-up 15H06657 (N.Y.), Grant-in-Aid for Young Scientists B 17K16401 (N.Y.), Grant-in-Aid for Scientific Research B 25281028 (to K.I. and N.Y. and M.I.O.) and Health Sciences Research Grants from the Ministry of Health, Labour and Welfare (K.I.). The funding bodies had no input in the design of the study, collection, analysis, and interpretation of data, or writing the manuscript.

\section{Authors' contributions}

NY and KI wrote the manuscript. NY performed the experiments. NY and KI conceived the experiments and analyzed the data. HT advised about in vivo experiments. SM and IH prepared samples. MIO, HT and $\mathrm{MN}$ correct the manuscript. All authors read and approved the final manuscript.

\section{Corresponding authors}

Correspondence to Naoki Yamamoto and Katsuhide Igarashi.

\section{Acknowledgements}


The authors thank our laboratory members in the member of Institute for Advanced Life Sciences for useful discussions on this topic.

\section{References}

1. Robertson KD. DNA methylation and human disease. Nat Rev Genet. 2005;6(8):597-610.

2. Mill J, Tang T, Kaminsky Z, Khare T, Yazdanpanah S, Bouchard L, et al. Epigenomic profiling reveals DNA-methylation changes associated with major psychosis. Am J Hum Genet. 2008;82(3):696-711.

3. Fuchikami M, Morinobu S, Segawa M, Okamoto Y, Yamawaki S, Ozaki N, et al. DNA methylation profiles of the brain-derived neurotrophic factor (BDNF) gene as a potent diagnostic biomarker in major depression. PLoS One. 2011;6(8):e23881.

4. Kordi-Tamandani DM, Sahranavard R, Torkamanzehi A. DNA methylation and expression profiles of the brain-derived neurotrophic factor (BDNF) and dopamine transporter (DAT1) genes in patients with schizophrenia. Mol Biol Rep. 2012;39(12):10889-93.

5. D'Addario C, Dell'Osso B, Palazzo MC, Benatti B, Lietti L, Cattaneo E, et al. Selective DNA methylation of BDNF promoter in bipolar disorder: differences among patients with BDI and BDII. Neuropsychopharmacology. 2012;37(7):1647-55.

6. D'Addario C, Dell'Osso B, Galimberti D, Palazzo MC, Benatti B, Di Francesco A, et al. Epigenetic modulation of BDNF gene in patients with major depressive disorder. Biol Psychiatry. 2013;73(2):e67.

7. Yamamoto N, Agata K, Nakashima K, Imamura T. Bidirectional promoters link cAMP signaling with irreversible differentiation through promoter-associated non-coding RNA (pancRNA) expression in PC12 cells. Nucleic Acids Res. 2016;44(11):5105-22.

8. Tomikawa J, Shimokawa H, Uesaka M, Yamamoto N, Mori Y, Tsukamura H, et al. Single-stranded noncoding RNAs mediate local epigenetic alterations at gene promoters in rat cell lines. J Biol Chem. 2011;286(40):34788-99.

9. Maeder ML, Angstman JF, Richardson ME, Linder SJ, Cascio VM, Tsai SQ, et al. Targeted DNA demethylation and activation of endogenous genes using programmable TALE-TET1 fusion proteins. Nat Biotechnol. 2013;31(12):1137-42.

10. Hamazaki N, Uesaka M, Nakashima K, Agata K, Imamura T. Gene activation-associated long noncoding RNAs function in mouse preimplantation development. Development. 2015;142(5):91020.

11. Amabile A, Migliara A, Capasso P, Biffı M, Cittaro D, Naldini L, et al. Inheritable Silencing of Endogenous Genes by Hit-and-Run Targeted Epigenetic Editing. Cell. 2016;167(1):219-32 e14.

12. Xu GL, Bestor TH. Cytosine methylation targetted to pre-determined sequences. Nat Genet. 1997;17(4):376-8.

13. Li F, Papworth M, Minczuk M, Rohde C, Zhang Y, Ragozin S, et al. Chimeric DNA methyltransferases target DNA methylation to specific DNA sequences and repress expression of target genes. Nucleic 
Acids Res. 2007;35(1):100-12.

14. Rivenbark AG, Stolzenburg S, Beltran AS, Yuan X, Rots MG, Strahl BD, et al. Epigenetic reprogramming of cancer cells via targeted DNA methylation. Epigenetics. 2012;7(4):350-60.

15. Siddique AN, Nunna S, Rajavelu A, Zhang Y, Jurkowska RZ, Reinhardt R, et al. Targeted methylation and gene silencing of VEGF-A in human cells by using a designed Dnmt3a-Dnmt3L single-chain fusion protein with increased DNA methylation activity. J Mol Biol. 2013;425(3):479-91.

16. Chen H, Kazemier HG, de Groote ML, Ruiters MH, Xu GL, Rots MG. Induced DNA demethylation by targeting Ten-Eleven Translocation 2 to the human ICAM-1 promoter. Nucleic Acids Res. 2014;42(3):1563-74.

17. Morita S, Noguchi H, Horii T, Nakabayashi K, Kimura M, Okamura K, et al. Targeted DNA demethylation in vivo using dCas9-peptide repeat and ScFv-TET1 catalytic domain fusions. Nat Biotechnol. 2016;34(10):1060-5.

18. Liu XS, Wu H, Ji X, Stelzer Y, Wu X, Czauderna S, et al. Editing DNA Methylation in the Mammalian Genome. Cell. 2016;167(1):233-47 e17.

19. Vojta A, Dobrinic P, Tadic V, Bockor L, Korac P, Julg B, et al. Repurposing the CRISPR-Cas9 system for targeted DNA methylation. Nucleic Acids Res. 2016;44(12):5615-28.

20. Stepper P, Kungulovski G, Jurkowska RZ, Chandra T, Krueger F, Reinhardt R, et al. Efficient targeted DNA methylation with chimeric dCas9-Dnmt3a-Dnmt3L methyltransferase. Nucleic Acids Res. 2017;45(4):1703-13.

21. Saunderson EA, Stepper P, Gomm JJ, Hoa L, Morgan A, Allen MD, et al. Hit-and-run epigenetic editing prevents senescence entry in primary breast cells from healthy donors. Nat Commun. 2017;8(1):1450.

22. Huang YH, Su J, Lei Y, Brunetti L, Gundry MC, Zhang X, et al. DNA epigenome editing using CRISPRCas SunTag-directed DNMT3A. Genome Biol. 2017;18(1):176.

23. Cali CP, Park DS, Lee EB. Targeted DNA methylation of neurodegenerative disease genes via homology directed repair. Nucleic Acids Res. 2019;47(22):11609-22.

24. Hanzawa N, Hashimoto K, Yuan X, Kawahori K, Tsujimoto K, Hamaguchi M, et al. Targeted DNA demethylation of the Fgf21 promoter by CRISPR/dCas9-mediated epigenome editing. Sci Rep. 2020;10(1):5181.

25. Liu XS, Wu H, Krzisch M, Wu X, Graef J, Muffat J, et al. Rescue of Fragile X Syndrome Neurons by DNA Methylation Editing of the FMR1 Gene. Cell. 2018;172(5):979-92 e6.

26. Doherty FC, Schaack JB, Sladek CD. Comparison of the efficacy of four viral vectors for transducing hypothalamic magnocellular neurosecretory neurons in the rat supraoptic nucleus. J Neurosci Methods. 2011;197(2):238-48.

27. Howarth JL, Lee YB, Uney JB. Using viral vectors as gene transfer tools (Cell Biology and Toxicology Special Issue: ETCS-UK 1 day meeting on genetic manipulation of cells). Cell Biol Toxicol. 2010;26(1):1-20. 
28. Lentz TB, Gray SJ, Samulski RJ. Viral vectors for gene delivery to the central nervous system. Neurobiol Dis. 2012;48(2):179-88.

29. Deyle DR, Russell DW. Adeno-associated virus vector integration. Curr Opin Mol Ther. 2009;11(4):4427.

30. Ginn SL, Amaya AK, Alexander IE, Edelstein M, Abedi MR. Gene therapy clinical trials worldwide to 2017: An update. J Gene Med. 2018;20(5):e3015.

31. Doudna JA, Charpentier E. Genome editing. The new frontier of genome engineering with CRISPRCas9. Science. 2014;346(6213):1258096.

32. Morita S, Horii T, Hatada I. Editing of DNA Methylation Using dCas9-Peptide Repeat and scFv-TET1 Catalytic Domain Fusions. Methods Mol Biol. 2018;1767:419-28.

33. Pflueger C, Tan D, Swain T, Nguyen T, Pflueger J, Nefzger C, et al. A modular dCas9-SunTag DNMT3A epigenome editing system overcomes pervasive off-target activity of direct fusion dCas9-DNMT3A constructs. Genome Res. 2018;28(8):1193-206.

34. Bernstein DL, Le Lay JE, Ruano EG, Kaestner KH. TALE-mediated epigenetic suppression of CDKN2A increases replication in human fibroblasts. J Clin Invest. 2015;125(5):1998-2006.

35. Bourc'his D, Xu GL, Lin CS, Bollman B, Bestor TH. Dnmt3L and the establishment of maternal genomic imprints. Science. 2001;294(5551):2536-9.

36. Jia D, Jurkowska RZ, Zhang X, Jeltsch A, Cheng X. Structure of Dnmt3a bound to Dnmt3L suggests a model for de novo DNA methylation. Nature. 2007;449(7159):248-51.

37. Hu L, Li Z, Cheng J, Rao Q, Gong W, Liu M, et al. Crystal structure of TET2-DNA complex: insight into TET-mediated 5mC oxidation. Cell. 2013;155(7):1545-55.

38. Bintu L, Yong J, Antebi YE, McCue K, Kazuki Y, Uno N, et al. Dynamics of epigenetic regulation at the single-cell level. Science. 2016;351(6274):720-4.

39. Hall J, Thomas KL, Everitt BJ. Rapid and selective induction of BDNF expression in the hippocampus during contextual learning. Nat Neurosci. 2000;3(6):533-5.

40. Chen WG, Chang Q, Lin Y, Meissner A, West AE, Griffith EC, et al. Derepression of BDNF transcription involves calcium-dependent phosphorylation of MeCP2. Science. 2003;302(5646):885-9.

41. Martinowich K, Hattori D, Wu H, Fouse S, He F, Hu Y, et al. DNA methylation-related chromatin remodeling in activity-dependent BDNF gene regulation. Science. 2003;302(5646):890-3.

42. Lubin FD, Roth TL, Sweatt JD. Epigenetic regulation of BDNF gene transcription in the consolidation of fear memory. J Neurosci. 2008;28(42):10576-86.

43. Mizuno K, Dempster E, Mill J, Giese KP. Long-lasting regulation of hippocampal Bdnf gene transcription after contextual fear conditioning. Genes Brain Behav. 2012;11(6):651-9.

44. Hofacker D, Broche J, Laistner L, Adam S, Bashtrykov P, Jeltsch A. Engineering of Effector Domains for Targeted DNA Methylation with Reduced Off-Target Effects. Int J Mol Sci. 2020;21(2).

45. Ishimaru N, Fukuchi M, Hirai A, Chiba Y, Tamura T, Takahashi N, et al. Differential epigenetic regulation of BDNF and NT-3 genes by trichostatin A and 5-aza-2'-deoxycytidine in Neuro-2a cells. 
Biochem Biophys Res Commun. 2010;394(1):173-7.

46. Nagahara AH, Merrill DA, Coppola G, Tsukada S, Schroeder BE, Shaked GM, et al. Neuroprotective effects of brain-derived neurotrophic factor in rodent and primate models of Alzheimer's disease. Nat Med. 2009;15(3):331-7.

47. Nagahara AH, Tuszynski MH. Potential therapeutic uses of BDNF in neurological and psychiatric disorders. Nat Rev Drug Discov. 2011;10(3):209-19.

48. Shimojo H, Ohtsuka T, Kageyama R. Oscillations in notch signaling regulate maintenance of neural progenitors. Neuron. 2008;58(1):52-64.

49. Imayoshi I, Isomura A, Harima Y, Kawaguchi K, Kori H, Miyachi H, et al. Oscillatory control of factors determining multipotency and fate in mouse neural progenitors. Science. 2013;342(6163):1203-8.

50. Jones MW, Errington ML, French PJ, Fine A, Bliss TV, Garel S, et al. A requirement for the immediate early gene Zif268 in the expression of late LTP and long-term memories. Nat Neurosci. 2001;4(3):289-96.

51. Hong EJ, McCord AE, Greenberg ME. A biological function for the neuronal activity-dependent component of Bdnf transcription in the development of cortical inhibition. Neuron. 2008;60(4):61024.

52. Ran FA, Cong L, Yan WX, Scott DA, Gootenberg JS, Kriz AJ, et al. In vivo genome editing using Staphylococcus aureus Cas9. Nature. 2015;520(7546):186-91.

53. Kearns NA, Genga RM, Enuameh MS, Garber M, Wolfe SA, Maehr R. Cas9 effector-mediated regulation of transcription and differentiation in human pluripotent stem cells. Development. 2014;141(1):219-23.

54. Heigwer F, Kerr G, Boutros M. E-CRISP: fast CRISPR target site identification. Nat Methods. 2014;11(2):122-3.

\section{Figures}


a

Vector \#1 ( 4.7 kb)

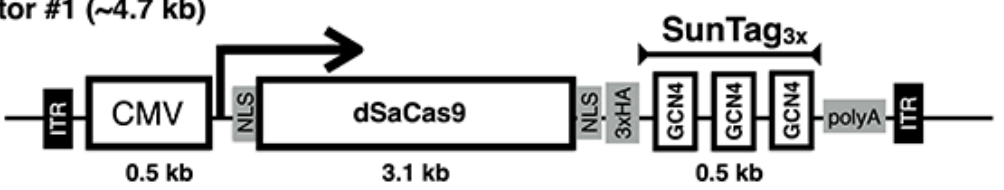

b

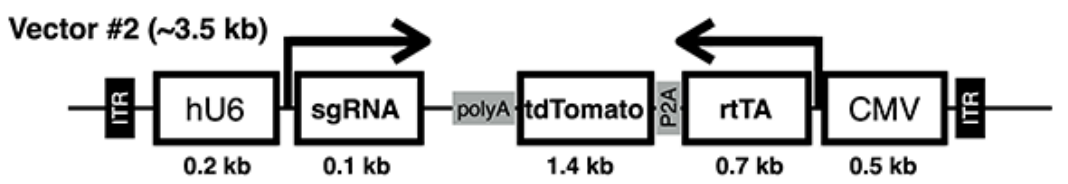

C Vector \#3 ( 3.5 kb)

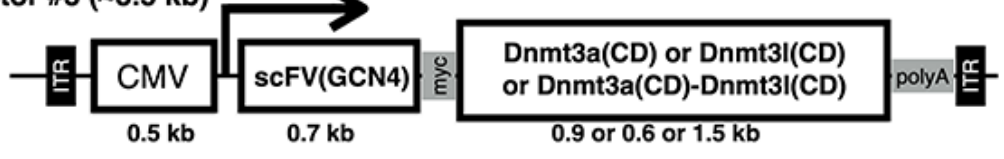

d

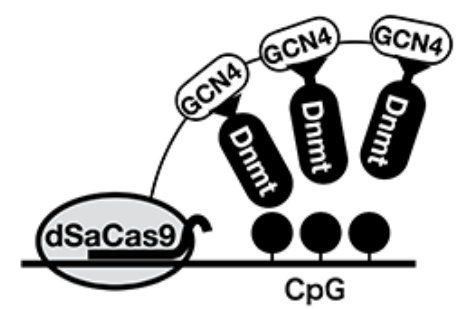

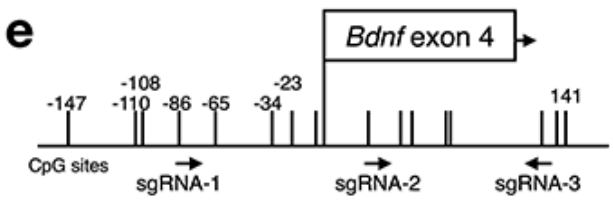

f

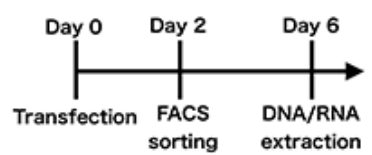

g

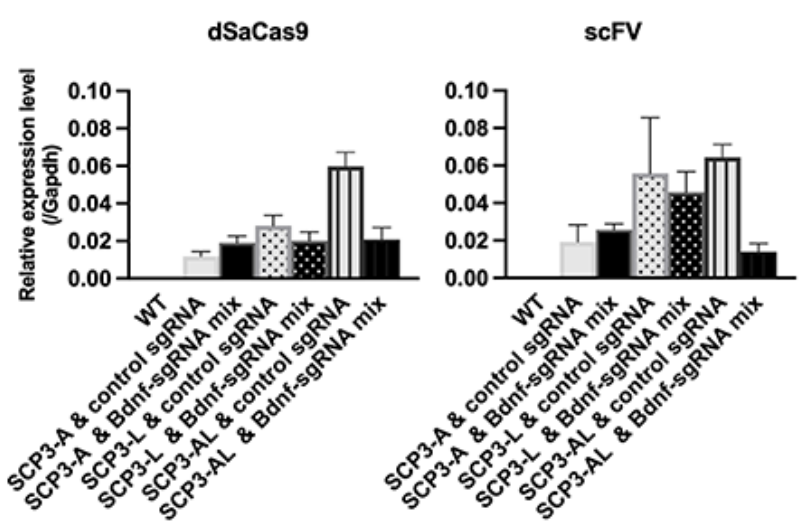

h
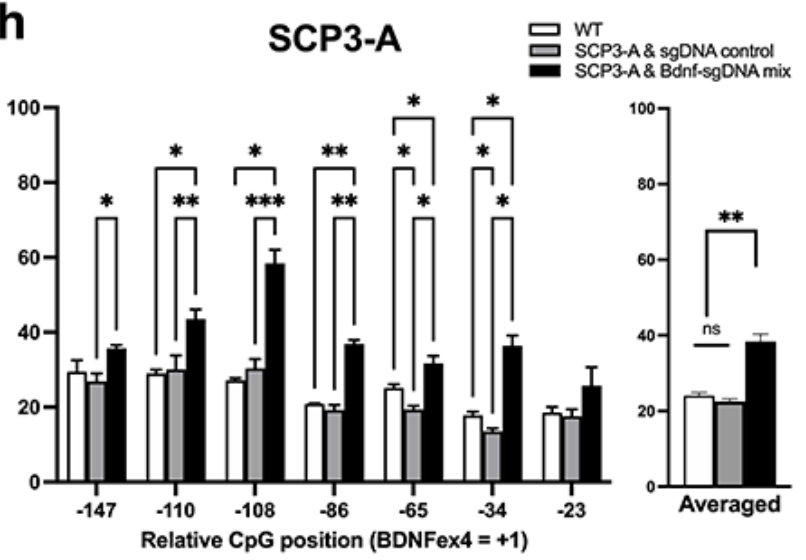

i

SCP3-L

다

D SCP3-L\& sgRNA control
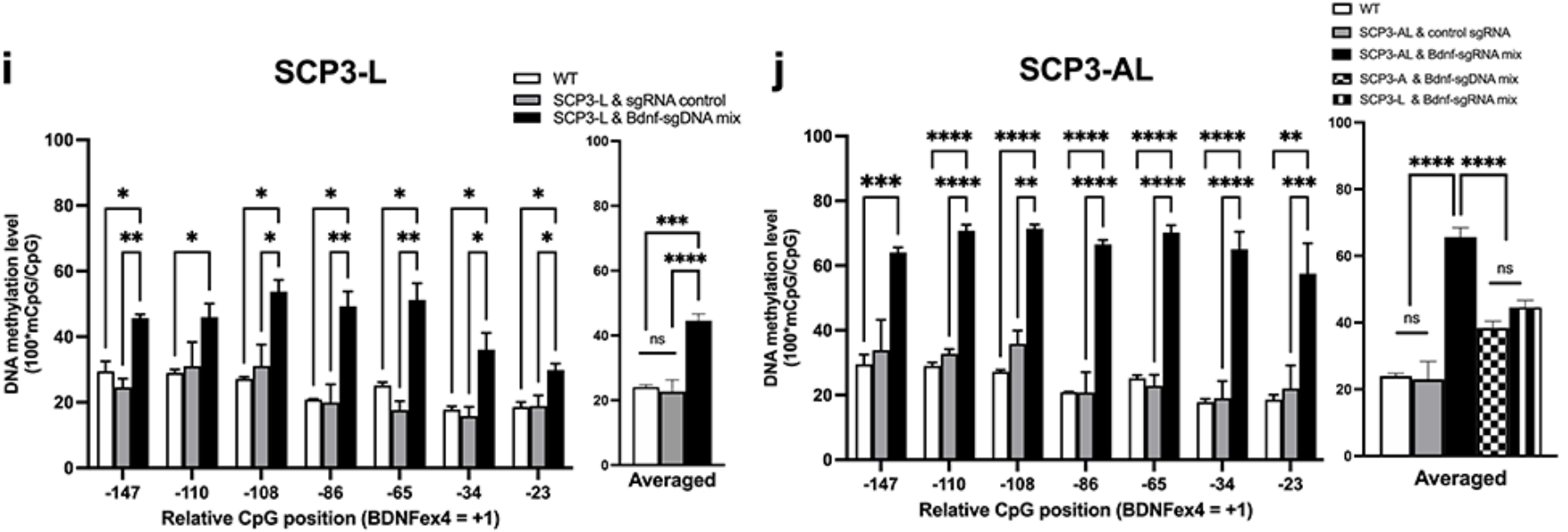

Figure 1

Targeted DNA methylation with the dSaCas9-SunTag and antibody-conjugated-Dnmts in vitro (a-c) Structure of AAV vector components. Size of transgene on each vector was shown. (d) Schematic representation of targeted DNA methylation with dSaCas9-SunTag system. (e) The mouse Gfap exon 4 locus. Positions of $\mathrm{CpG}$ sites are numbered relative to TSS of the Bdnf exon 4 and indicated by vertical black bars. The locations of the sgRNA targeting sequence are indicated by black arrows. (f) The 
experimental scheme in this experiment. (g) RT-qPCR analysis of dSaCas9 and scFv. (h-k) Left panel: The percent of DNA methylation across individual CpGs on the Bdnf exon 4 as determined by bisulfite pyrosequencing analysis Right panel: The averaged DNA methylation level shown in left panel. (g-j) Values are mean \pm SD from ( $w t: n=3$, SCP3-A and SCL3-L $: n=3$, SCP3-Dnmt3AL $: n=6$, independent transfections). Statistical analysis were performed using two-way repeated measure ANOVA with Tukey's multiple comparison in ( $h$, i left), mixed-effects analysis with Tukey's multiple comparison in ( $\mathrm{l}$ left) and one-way repeated measure ANOVA with Tukey's multiple comparison in ( $h, i$ and $j$ right). ns: not significant; $*<0.05, * *<0.01, * * *<0.001, * \star * *<0.0001$. 
a

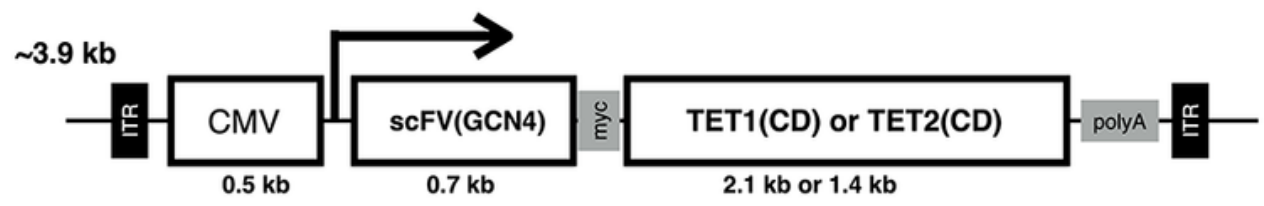

b

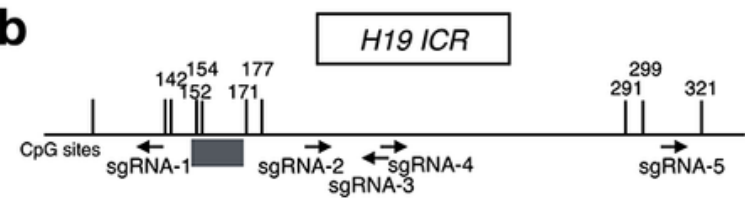

C

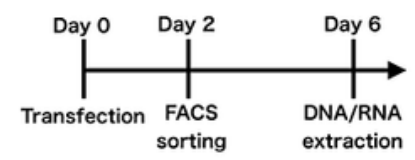

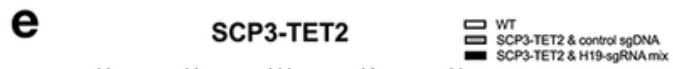
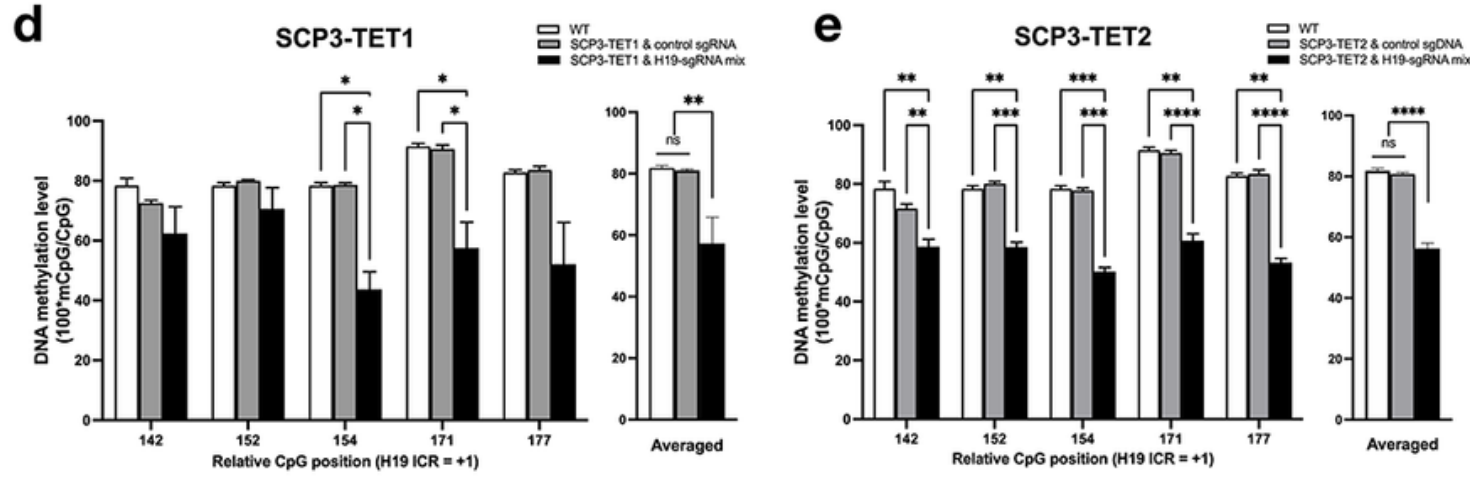

f
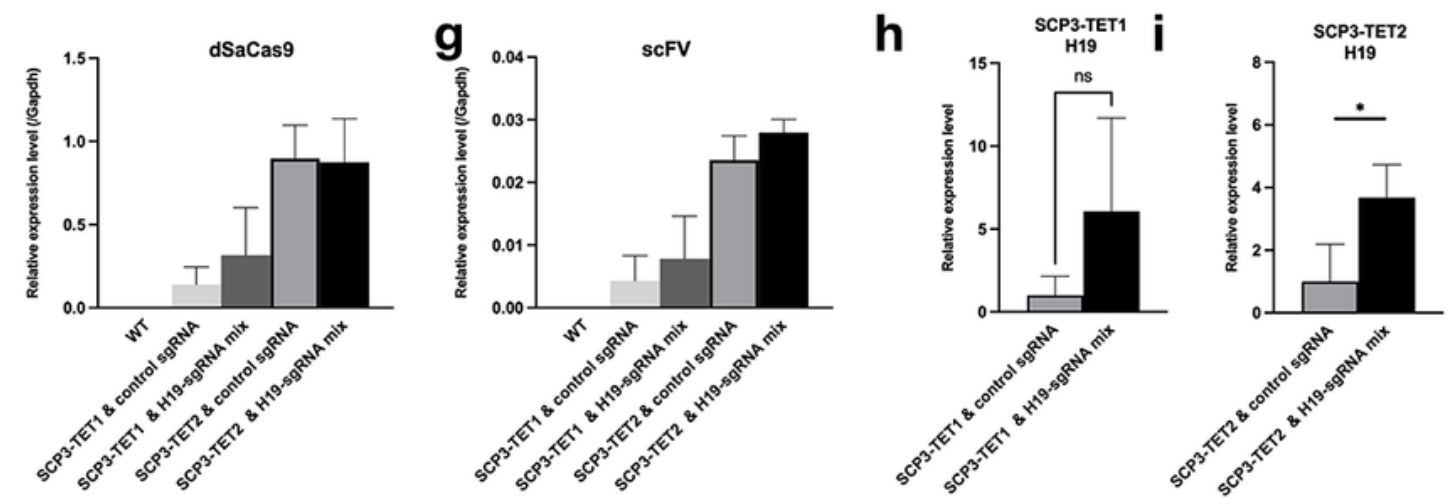

Figure 2

Targeted DNA demethylation with the dSaCas9-SunTag and antibody-conjugated-TET1 or TET2 in vitro (a) Structure of AAV vector expressing scFv-TET1CD or scFv-TET2CD. (b) The mouse H19 ICR locus. CTCF-binding sites indicated by grey box. Positions of CpG sites are numbered relative to edge of $\mathrm{H} 19$ ICR and indicated by vertical black bars. The locations of the s-sgRNA targeting sequence are indicated by black arrows. (c) The experimental scheme in this experiment. (d, e) Left panel: The percent of DNA 
methylation across individual $\mathrm{CpGs}$ on $\mathrm{H} 19$ ICR as determined by bisulfite pyrosequencing analysis. Right panel: The averaged DNA methylation level shown in left panel. (f, g) RT-qPCR analysis of dSaCas9 and scFv. $(h, i)$ Fold change in the expression levels of the H19 genes. Mean expression level in control-sgRNA transfected sample was set to 1 . ( $(\mathrm{d}-\mathrm{g}) \mathrm{n}=3$ for $\mathrm{wt}, \mathrm{n}=3$ independent transfections for SCP3-TET1, $\mathrm{n}=4$ independent transfections with SCP3-TET2. Statistical analysis were performed using two-way repeated measure ANOVA with Tukey's multiple comparison in ( $\mathrm{d}$ and e left), one-way repeated measure ANOVA with Tukey's multiple comparison in ( $\mathrm{d}$ and e right) and Student's two-tailed t-tests in ( $\mathrm{h}, \mathrm{i})$.
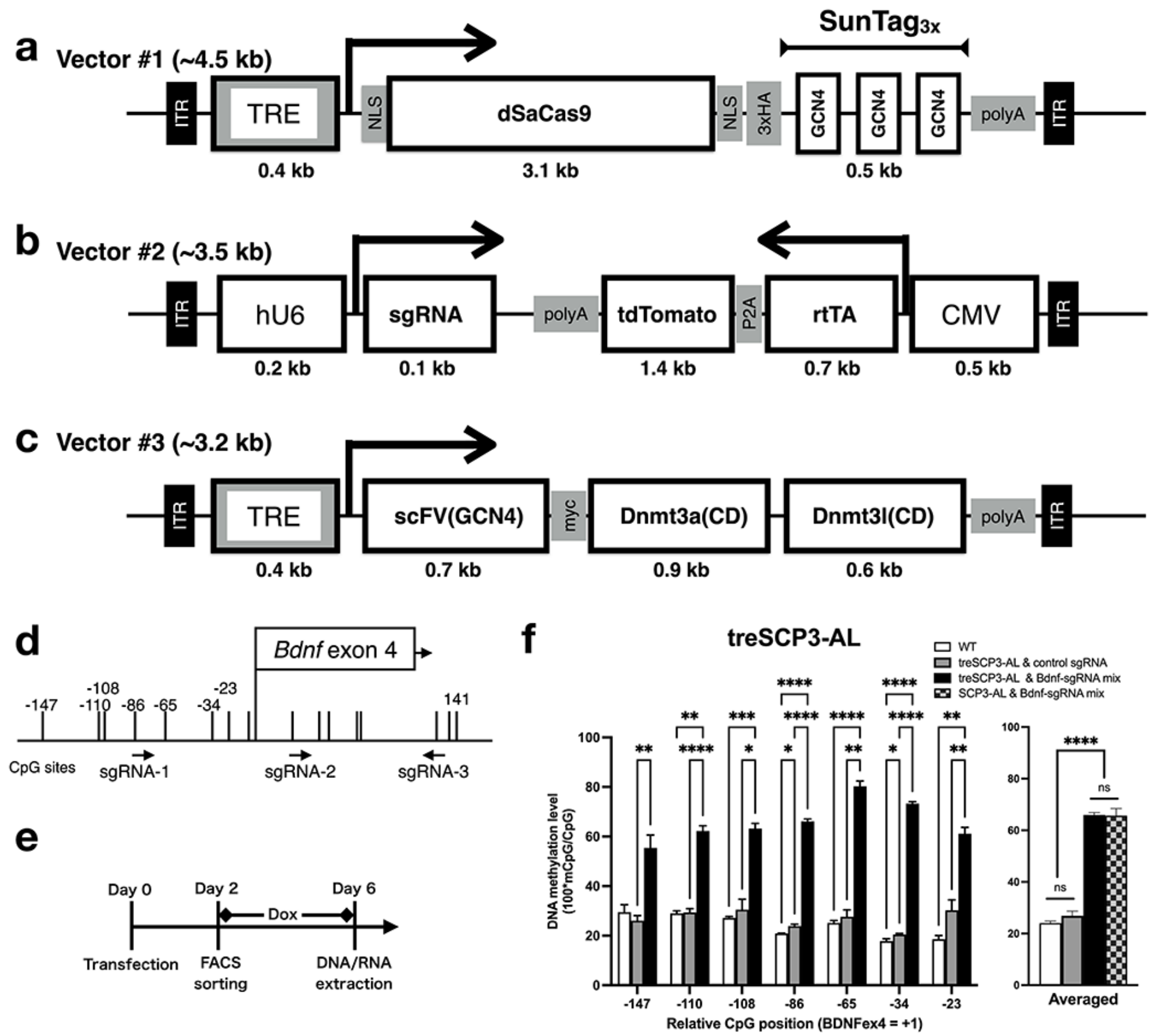

Figure 3 
Dox-inducible in vitro targeted DNA methylation with the dSaCas9-SunTag and antibody-conjugatedDnmt3AL in vitro (a-c) Structure of AAV vectors for the Dox-inducible SCP3-AL system. Size of transgene on each vector was shown. (d) The mouse Gfap exon 4 locus. (e) The experimental scheme in this experiment. (f) Left panel: The percent of DNA methylation across individual CpGs on the Bdnf exon 4 as determined by bisulfite pyrosequencing analysis. Right panel: The averaged DNA methylation level shown in left panel. (f) $n=3$ for wt, $n=3$ independent transfections for treSCP3-AL. Statistical analysis were performed using two-way repeated measure ANOVA with Tukey's multiple comparison in ( $\mathrm{fleft}$ ) and oneway repeated measure ANOVA with Tukey's multiple comparison in ( $\mathrm{f}$ right). 
a

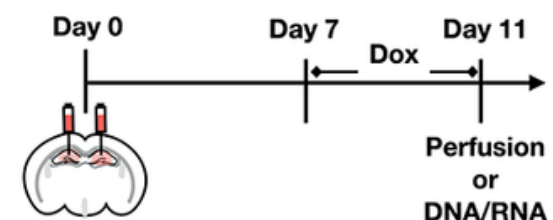

AAV injection to HPC

- TRE-dSaCas9-SunTag $3 x$

- U6-sgDNA;CMV-rtTA-tdTomato

DNA/RNA

Extraction
- TRE-scFV-Dnmt3AL

b

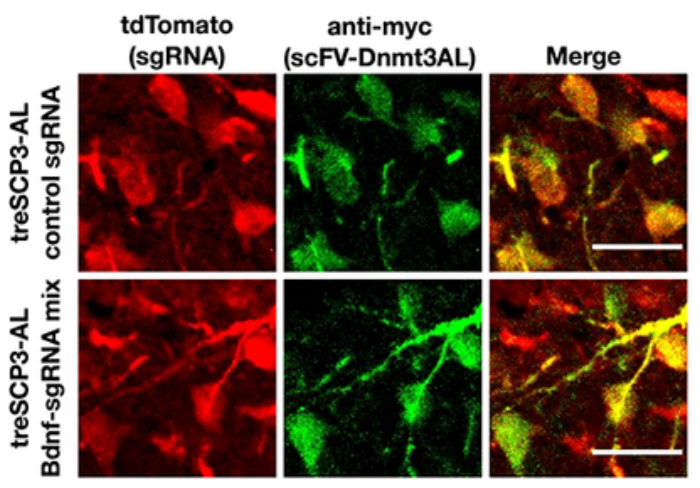

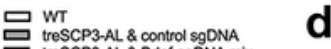

$$
\text { U6-sgDNA,CMV-rtTA-tdTomato }
$$

c

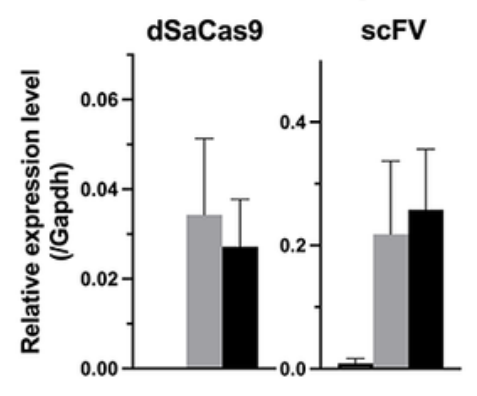

e
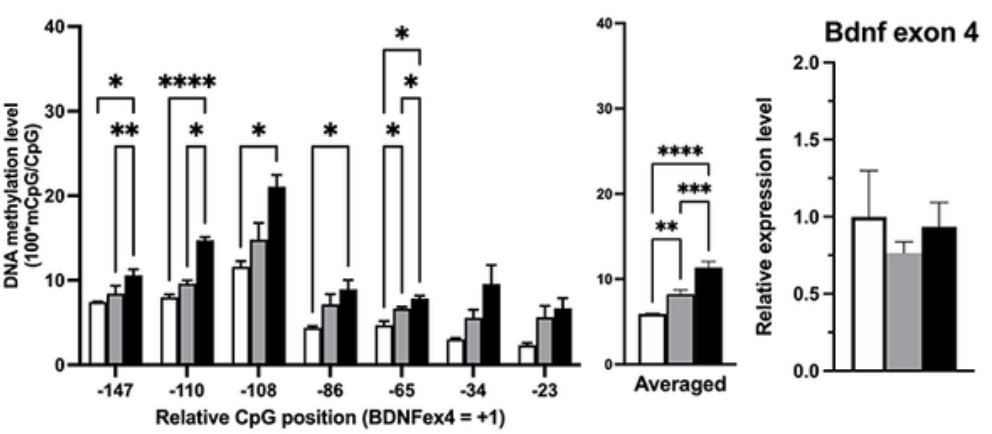

$\mathbf{f}$

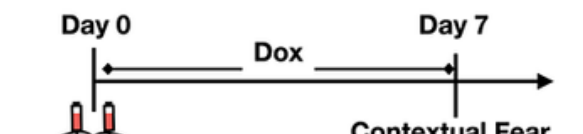

h

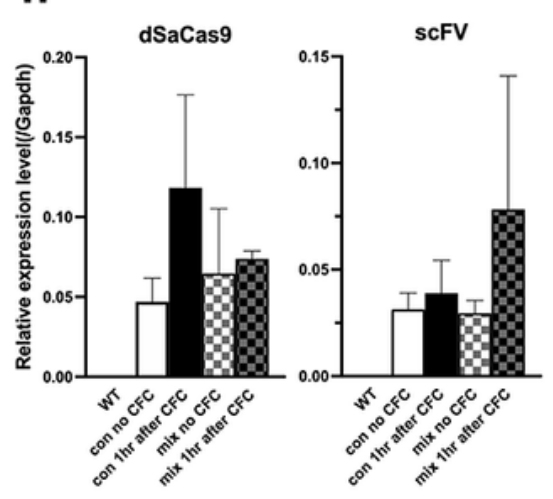

i

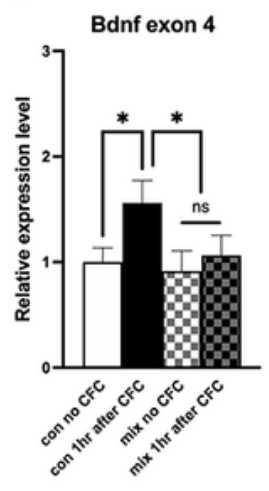

\section{Figure 4}

AAV-mediated in vivo targeted DNA methylation with Dox-inducible the SCP3-AL (a) The experimental scheme in this experiment. After bilateral AAV injection to HPC, Dox was administered in drinking water. (b) The tdTomato-labelled cell bodies (red) on dorsal HPC were immunostained with anti-myc (green). Scale bar: $30 \mu \mathrm{m}$. (c and h) RT-qPCR analysis of dSaCas9 and scFv. (d) Left panel: The percent of DNA methylation across individual CpGs on the Bdnf exon 4 as determined by bisulfite pyrosequencing 
analysis. Right panel: The averaged DNA methylation level shown in left panel. (e) Fold change in the expression levels of the Bdnf exon 4. Mean expression level in WT sample was set to 1. (f) The experimental scheme in this experiment. Half of AAV-injected animals were subjected to 2 times of CFC and sacrificed $1 \mathrm{hr}$ later. The remaining half was sacrificed without CFC (no CFC). (g) Freezing rate of animal during CFCs. Red vertical line indicate the timing delivered a foot shock. Mean + SEM. (i) Fold change in the expression levels of the Bdnf exon 4. Mean expression level in control-sgRNA transfected and no-CFC sample was set to 1. (c, e) wt: $n=3$, control-sgRNA: $n=4, B d n f-m i x: n=5$. (d and g-i) $n=3$. Statistical analyses were performed using Two-way repeated measure ANOVA with Tukey's multiple comparison in (e left) or one-way repeated measure ANOVA with Tukey's multiple comparison in (e right). 